\title{
Wireless Physiological Parameter Monitoring and Recording System
}

\author{
M. Manojprabu \\ M.E, Embedded System Technologies University college of Engineering BIT campus, Trichy
}

\begin{abstract}
The design and development of a Zigbee smart non-invasive wearable physiological parameters monitoring device has been developed and reported in this paper. The system can be used to monitor physiological parameters, such as temperature and heart rate, of a human subject. The system consists of an electronic device which is worn on the wrist and finger, by an at-risk person. Using several sensors to measure different vital signs, the person is wirelessly monitored within his own home. An impact sensor has been used to detect falls. The device detects if a person is medically distressed and sends an alarm to a receiver unit that is connected to a computer. This sets off an alarm, allowing help to be provided to the user. The device is battery powered for use outdoors. The device can be easily adapted to monitor athletes and infants. The low cost of the device will help to lower the cost of home monitoring of patients recovering from illness. A prototype of the device has been fabricated and extensively tested with very good results.
\end{abstract}

\section{Introduction}

IN Recent Times, wireless sensors and sensor networks have become a great interest to research, scientific and technological community. Though sensor networks have been in place for more than a few decades now, the wireless domain has opened up a whole new application space of sensors. Wireless sensors and sensor networks are different from traditional wireless networks as well computer networks and, therefore, pose more challenges to solve such as limited energy, restricted life time, etc. [1].

Wireless sensing units integrate wireless communications and mobile computing with transducers to deliver a sensor platform which is inexpensive to install in numerous applications. Indeed, co-locating computational power and radio frequency (RF) communication within the sensor unit itself is a distinct feature of wireless sensing. Today, the progress in science and technology offers miniaturization, speed, intelligence, sophistication, and new materials at lower cost, resulting in the development of various high-performance smart sensing system. Many new research is focused at improving quality of human life in terms of health [2] by designing and fabricating sensors which are either in direct contact with the human body (invasive) or indirectly (noninvasive). One of the reasons for more development in this area is the global population and rise in ageing population [3], one statistic provided by the U.S. Department of Health that by 2050 over $20 \%$ of the world's population will be above 65 years of age. This results in a requirement for medical care, which is expensive for long-term monitoring and long waiting lists for consultations with health professionals. The cost of hospitalization is ever increasing, so is the cost of rehabilitation after a major illness or surgery. Hospitals are looking at sending people back as soon as possible to recoup at home. During this recovery period, several physiological parameters need to be continuously measured. Hence, telemedicine and remote monitoring of patients at home are gaining added importance and urgency [4]-[6]. Patients are being monitored using a network of wireless sensors [7]. Many elderly people dread the idea of being forced to live with their adult children, or in a rest home or in other sheltered living arrangement. They want to live independently and keep control of their own lives. Yet at the same time they know there is a high risk of injury or even death because of a fall or stroke. Such people need to be monitored continuously and provided with immediate medical help and attention when required. We seek to come up with solutions, which help to remove anxiety. As a result, there is a need for an accurate, flexible, noninvasive, comfortable, reliable, and low-cost monitoring unit that unites all these demands.

A system to monitor the overall health of welfare facility residents, who need constant care, has been reported in [5]-[8]. This system [8] has been designed with wireless sensors, wireless repeaters and a host computer. The system consists of a piezoelectric sensor, a two-axis accelerometer, a microcontroller, and a lowpower transceiver. It records respiration activity and indicators of posture for 24 hours. These data are transmitted to the wireless repeater by the transceiver. The wireless repeaters, which are installed throughout the welfare facility, send data, including the repeater's ID, to the host computer. The ID is used to detect the resident's location in the welfare facility. The host computer stores the data, which can be used to analyze the resident's overall health condition. When the resident is in an emergency situation, such as falling or in an inactive state for more that the allotted time, the host computer automatically alerts the situation to the care staff by an alarm sound and also by mobile phone. After researching work related to fall detection, falls are the most 
widespread domestic accidents among the elderly. Furthermore, it frequently happens that elderly people who have previously experienced a fall fear a new fall and sink gradually into inactivity. Due to less mobility it leads progressively to an increase in the risk of a fall [17]. Literature review reveals that reliable fall detection based raw sensor data is much discussed in literature and requires algorithm development of wide scope based on deeper knowledge of specific [18] application principles as outlined in and to monitor a range of human movement. However, all reported systems are relatively expensive and the cost depends on the number of sensors used. So, there is an effort to develop the home monitoring system using optimum number of sensors [20].

These facts show an increasing demand for long-term health monitoring which is affordable, continuous, and unobtrusive [9], which will result in considerable impact on annual medical costs [2] and health management [10], [19]. Wearable systems for continuous health monitoring are a key technology in helping the transition to more practical and affordable healthcare. It not only allows the user to closely monitor changes in her or his physiological parameters but also provides feedback to help maintain an optimal health status.

Currently, there are monitoring products in the market that are aimed to provide emergency assistance to senior citizens, rehabilitation patients, and medically or physically challenged individuals, but these have limitations. St. John's and Medic Alert's Lifelink ${ }^{\mathrm{TM}}$ [12] allows the user to set off an alarm manually if they are under medical stress, which will then dial designated contact phone numbers. The fundamental problem with this system is that when medical emergencies happen to the user, they are often unconscious and unable to press an "emergency alert button." There is no product on the market which does not require manual activation of the alarm and monitors a user's vital signs smartly, though research is currently undergoing [22]. This is the novel design goal of the work presented in this paper.

The reported device consists of a wrist strap and a finger ring (circuitry). This allows the sensors to be mounted around the wrist and finger and the 8051 microcontroller unit connected via ribbon cable. In Section II, we present the complete system overview. All the sensors are explained in Section III. The hardware details are in Section IV and the algorithms in Section V. The prototype and test results are discussed in Section VI. This paper ends with a discussion on future developments

\section{System Overview}

Fig. 1 shows the functional block diagram of the system hardware. The system has been designed to take several inputs to measure physiological parameters of human such as temperature, heart rate, and detection of any fall. The inputs from the sensors are integrated and processed. The results are sent through the XBee Module to a host computer, which stores the data into an Access Database. The values can then be displayed on the Graphical User Interface (GUI) running on a computer. If it is inferred that the person is medically distressed, an alarm may be generated. The program is a user interface, allowing a report on the current status of the individual. Once the user has connected to the receiver unit, data is automatically updated.

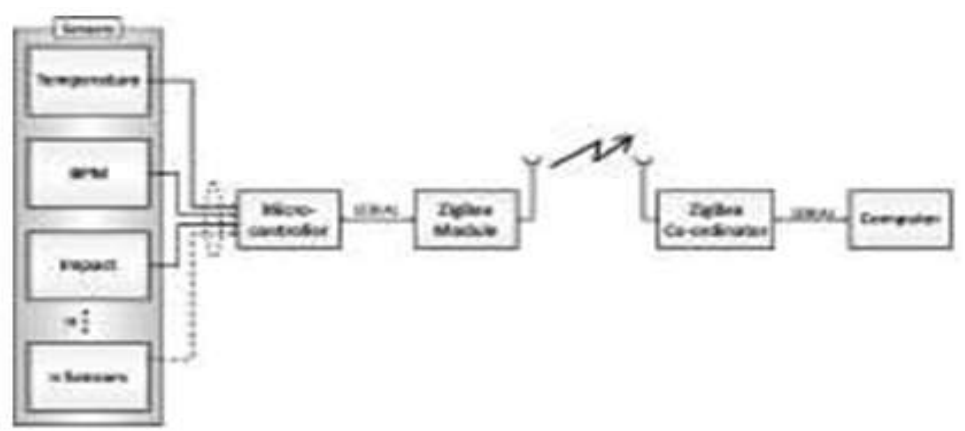

Fig 1. Functional block diagram of system hardware.

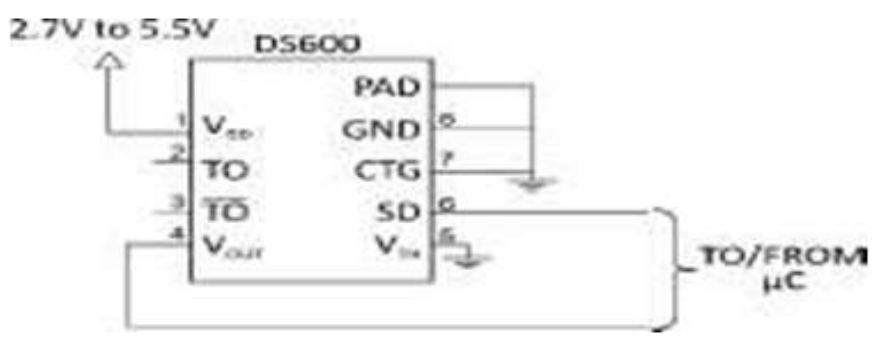

Fig. 2. Circuit application of DS600 temperature sensor. 
On the screen. Beat per minute (BPM), body temperature, and impact (in both axes) are given on the display. The data are also plotted on a time graph which can be customized to show data received from any of the Ensors.

The design is modular which makes it rather easy and straight forward to add extra sensors for measuring and monitoring other parameters. The hardware blocks are explained in full details in a later section.

\section{Details of the Sensing System}

The current version of the system consists of three sensors: a temperature sensor, heart rate sensor, and an impact sensor. Temperature sensor circuitry used in the design generates analog voltage which is fed to the ADC (Analog-to-Digital) inputs of the micro-controller. The ADC input is time-multiplexed and sampled at different rates. The description of individual sensors follows.

\section{A. Temperature Sensor}

The skin temperature measurement is done using an integrated circuit, the DS600 temperature sensor produced by MAXIM - Dallas Semiconductor [11]. The Sensor gives an analog output depending on the measured temperature. This voltage has to be measured by the microcontroller using a 12 bit Analog-to-Digital converter (ADC). Fig. 2 shows the circuit application of DS600 IC, used as temperature sensor This sensor is mounted within the wrist strap, positioned in such a way that it is in contact with the skin, allowing it to measure the external temperature of the skin. From the skin temperature, the body temperature is estimated. There can be different methods to estimate the exact body temperature from skin temperature [23], but with a rough estimation usually the body temperature is $5.1 \mathrm{C}$ higher than skin temperature when the body temperature is measured at the ear by the National DM-T2-A thermometer used by a general practitioner compared to the skin temperature

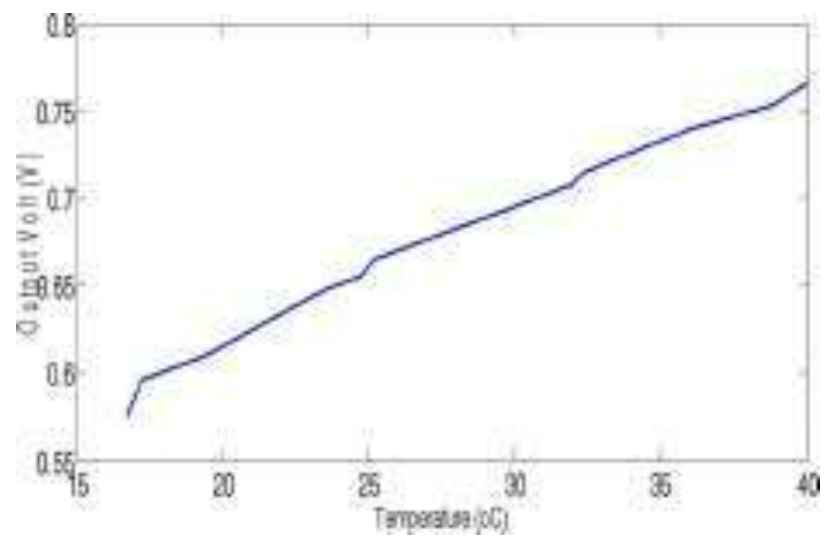

Fig. 3. Measured transfer characteristics of DS600.

Measured at the wrist. Because an exact measurement of body temperature is not required, this method is suitable. Rather, relative changes are monitored within set threshold, which sets off the alarm. This allows the device to detect changes in body temperature that could indicate the patient is undergoing any of the following conditions: trauma, injury, heart attack, stroke, heat exhaustion, and burns [14].

The IC has an accuracy of $\pm 0.5 \mathrm{C}$ and a linear output with

$6.45 \mathrm{C}$ and an offset of $509 \mathrm{mV}$ of DS600 IC factory calibration [11], which is shown by the experimental transfer characteristics, as in Fig. 3.

The Output of the ADC has to be converted into the right value. The ADC-value is first compared with the Reference Voltage of $2.4 \mathrm{~V}$ (1) and then with the characteristic of the DS 600 to get the Value for the

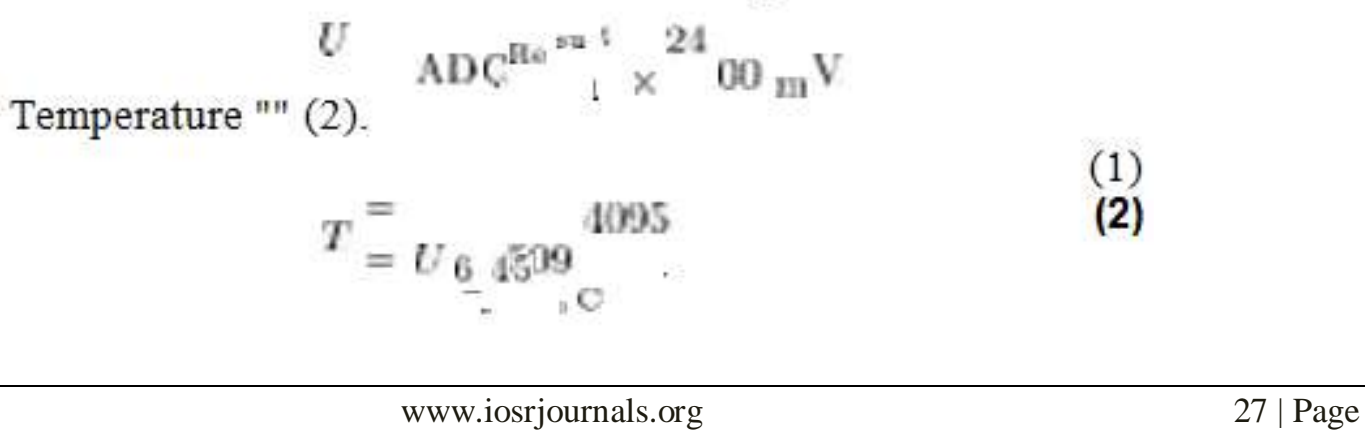


The sensor has been used for measurement of body temperature at wrist, upper arm and neck for many males and females and only two results are shown here. Fig. 4 shows the comparison of temperature measured by the developed temperature sensor with respect to a thermometer (reference temperature). The temperature at three different positions (wrist, neck and upper arm) was measured for three male and three female persons. The temperatures were measured at different times with varying ambient conditions. Fig. 5 shows the variations in temperature measured with respect to different positions. The accuracy of the measurement is shown in Fig. 5 and is seen that at steady state the error is within $0.5 \mathrm{C}$.

\section{B. Heart Rate Sensor}

A custom heart rate sensor was designed to read the patient's beats per minute (bpm). The designed sensor is very small and inexpensive. The technique used to measure the heart rate is based on near-infrared spectroscopy (NIR). NIR involves using light in the wavelength of 700-900 $\mathrm{nm}$ to measure blood volume. At these wavelengths most tissues do not absorb light - other than haemoglobin (which is what we are interested

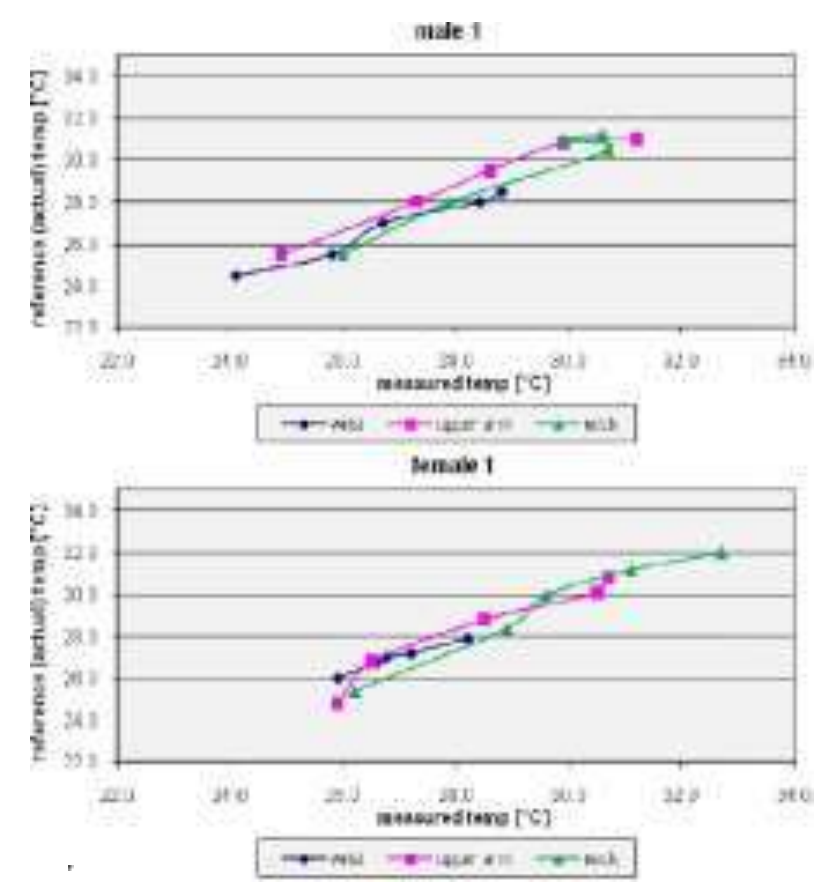

Fig. 4. The measured temperature of one male and one female.

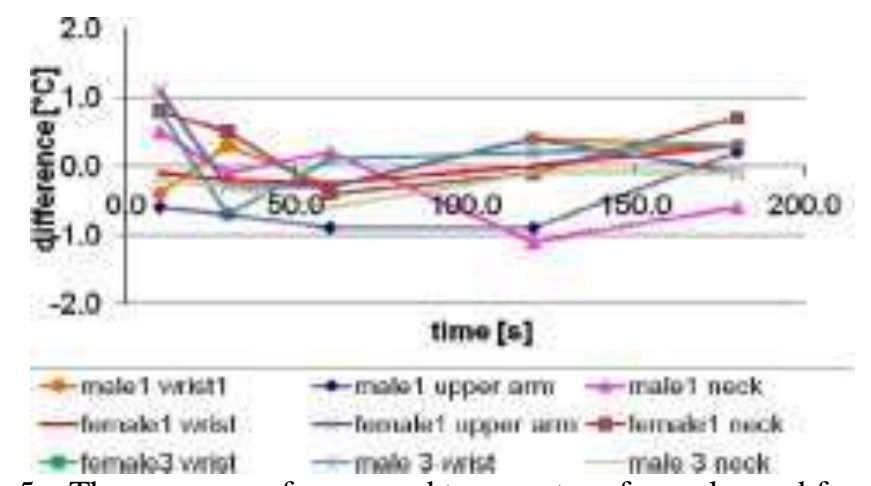

Fig. 5. The accuracy of measured temperature for males and females. 


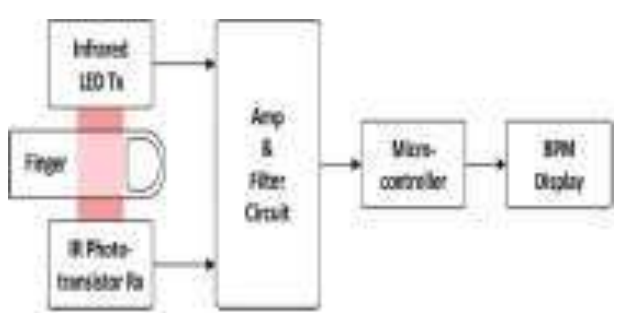

Fig. 6. Functional block diagram of the heart rate sensor [21].

This allowed for designing a noninvasive and low cost method of measuring the pulse. A silicon phototransistor, moulded into a flat side-facing package, and a GaAs Infrared Emitting Diode were used in the sensor.

Fig. 6 shows the functional block diagram of the heart rate sensor. The amount of light that was detected by the phototransistor varied with the patient's heart pulse, as the amount of absorbed IR light changed with the flow of blood, which is directly linked to the heart rate. This signal was then amplified, filtered, and sent to the microcontroller to be analyzed. The heart rate

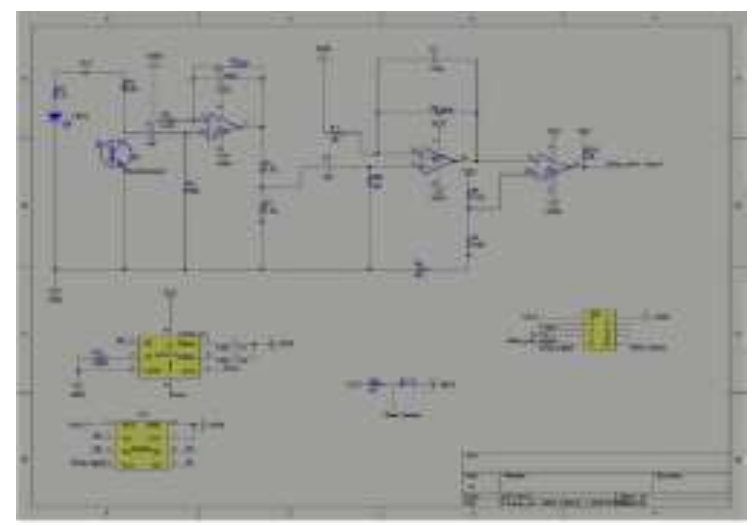

Fig. 7. The circuit schematic of heart rate measurement circuit.

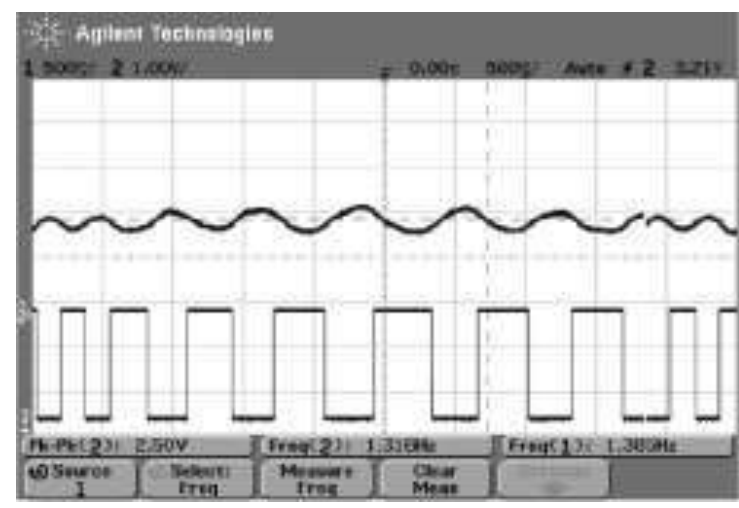

Fig. 8. The heart rate signal at the collector of the photo-transistor and at the output (Heart_Rate_signal).

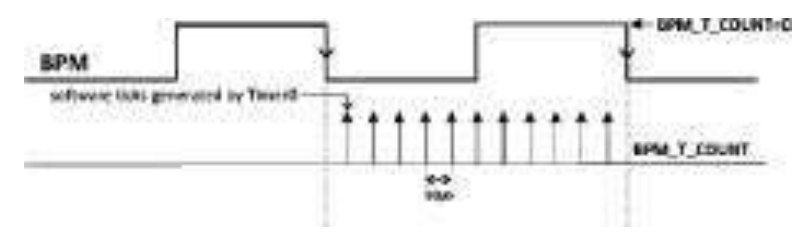

Fig. 9. Heart rate measurement algorithm. 


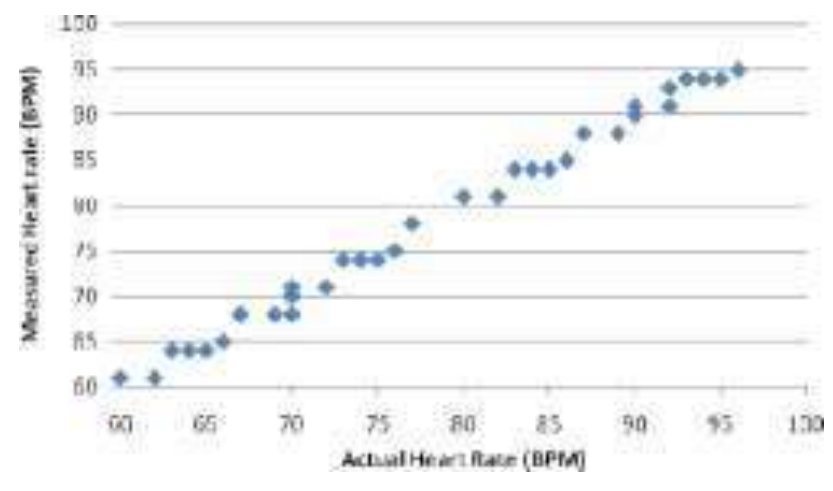

Fig. 10. The comparison of heart rate measurement.

Form of pulses is interfaced with microcontroller through its digital port for further processing. The waveforms at the collector of the photo-transistor and at the final point (Heart_rate_signal) are shown in Fig. 8. The heart rate is measured by using the hardware interrupt facility of the microcontroller. The heart rate as is shown in Fig. 8 is a square wave pulse of varying duty ration. The time period of the wave is measured using the Timer 0 and in combination with hardware interrupt. The measurement algorithm is explained with the help of Fig. 9. The Timer 0 generates a tick pulse at every 10 . The total tick count in one period (BPM_T_COUNT) is measured. The frequency is then calculated using the equation Sensor was mounted in the finger ring as this position proved to give the best response.

The hardware was built in two separate blocks. A sensor PCB was designed to house the temperature sensor, accelerometer and the connections for the NIR emitter and detector. The temperature, heart rate and impact sensors output was fed directly to the microcontroller through header pin-ribbon cable connection. The microcontroller was mounted onto a separate PCB which also had the ZigBee module connection. Fig. 7 shows the circuit schematics.

The sensor after filtering provided a clean wave that when observed on an oscilloscope confirmed that the sensor was correctly measuring the patients pulse. To get the best and most accurate results with the heart rate sensor we chose to measure the pulse at the finger tip like commercial device do. Nevertheless, it was checked for working on the wrist and the finger, too. The signal (analog) originally was too small to detect, and without amplification proved to be too noisy to extract the heart rate. Because of this, operational amplifiers were used to extract the heart rate signal. After amplifying, the signal was fed to comparator, resulting output in the form of pulses. The signal in the

Fig. 10 shows the comparison of heart rate measurement with a standard instrument and it is seen that the maximum error is $2 \mathrm{bpm}$.

It was observed that while measuring heart rate with the sensor developed, it showed more accuracy, as shown in Fig. 10, in terms of continuous monitoring in comparison to a sport watch (WR30M) with a similar sensor Was used as reference.
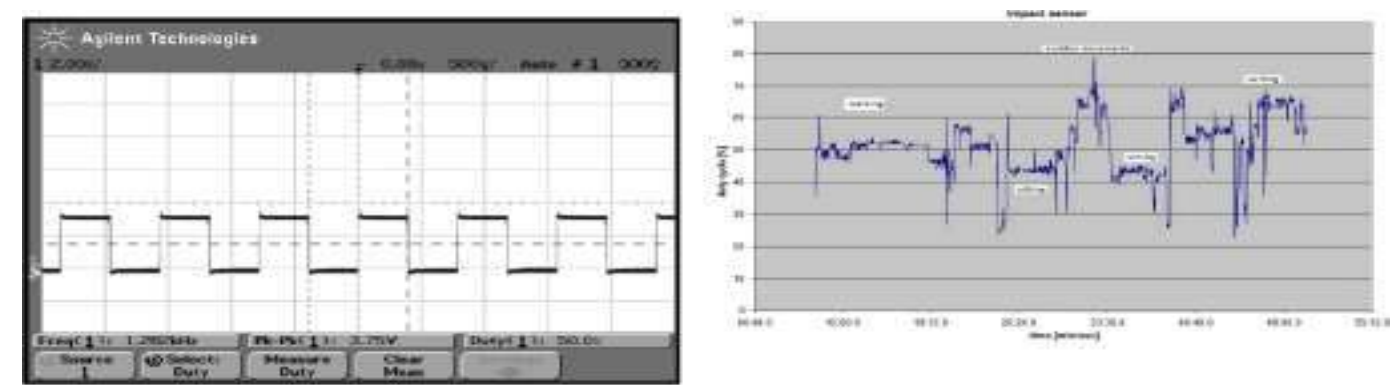

Fig. 11. Typical waveform of impact sensor in oscilloscope. Fig. 12. The typical results of the impact sensor along one (Y) axis.

$+/-2$. This was fitted into the wrist strap. This device provided a digital voltage, the amplitude of which was directly proportional to acceleration. The acceleration can be determined by measuring the length of the positive pulse width ( $\mathrm{t} 1$ ) and the period ( $\mathrm{t} 2$ ) see Fig. 8. The nominal transfer function of the ADXL213 is: 
Sensitivity = Minimum magnitude of input signal required to produce a specified output signal having a specified signal-to-noise ratio, or other specified criteria

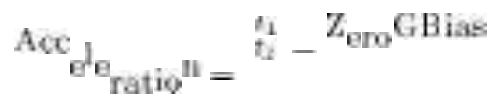

Where in the case of the ADXL213

Zero g Bias $=50 \%$ nominal;

Sensitivity $=30 \% / \mathrm{g}$ nominal.

The outputs are digital signals whose duty cycles (ratio of pulse width to period) are proportional to acceleration. This duty cycle can be directly measured using microcontroller.

Software algorithms were used to detect sharp impacts, while allowing slower movements, such as walking, to be ignored. The purpose of this sensor was to detect sudden impacts that could indicate the patient had fallen over. Fig. 11 shows the typical waveform of the impact sensor in oscilloscope, when user is standing in normal position, value of duty cycle is around $50 \%$.

The signal from the impact sensors is measured in a very similar way like the heart rate signal. In this case, both the measurements of the duty ratio is important. The impact sensor has been used for different conditions and the Fig. 12 shows the typical results. In this project, only one axis () is used to analyze movements.

The output of the accelerometer was tested with walking and simulated falling. The output of the accelerometer was tested with walking, everyday movements like sitting, standing, writing, etc., and simulated falls. The results showed the difference was simple to detect and proved the accuracy of the algorithm. Fig. 13 shows the impact sensor output. On analysis of the impacts it shows the difference in duty cycle during falls (under controlled conditions)

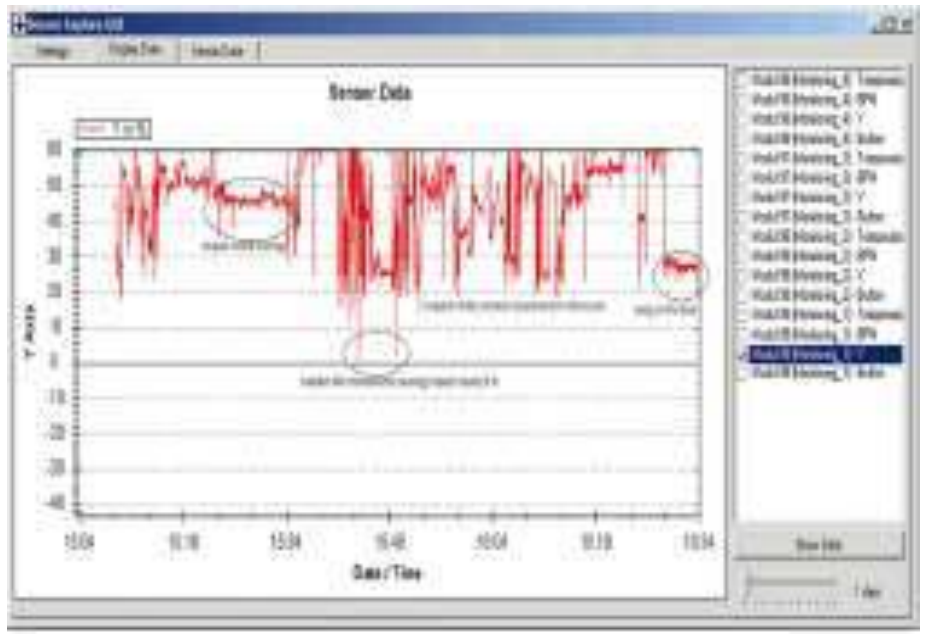

Fig. 13. Impact sensor output for walking and a fall.

\section{Microcontroller Interfacing And Communication}

The microcontroller used in the wrist strap unit is the Silicon Laboratories, Inc. C8051F020. The Microcontroller is programmed using " $\mathrm{C}$ " language for the operation of the above mentioned tasks in this project. This takes inputs from the sensors in the form of analog and digital voltages. Each sensor has a dedicated channel, ADC for temperature sensor, digital port0 for heart rate and impact sensor which is multiplexed by the microcontroller. Each sensor's signal is sampled at a predefined rate, through interrupt-driven algorithms.

\section{A. Communication}

Communication between the wrist units and the receiver unit is wireless. The data measured by the sensors is saved by building a network between the sensors and to set up a computer receiving and storing the values. For the communication ZigBee modules were used, powered by the Silabs C8051F020 microcontroller and transmitted in the unlicensed $2.4 \mathrm{GHz}$ frequency band. These provide a wide range and a couple of lowpower modes, which could be used to reduce the current consumption of the circuit. In addition, the network- 
setup is easy and fast, so that an extension of new units is possible without problems. Fig. 14 shows the connection overview of various sensor units, wirelessly.

The reason this microcontroller was chosen, was because of its low-power consumption, and built-in UART function for serial transmission of data to ZigBee module for wireless trans-

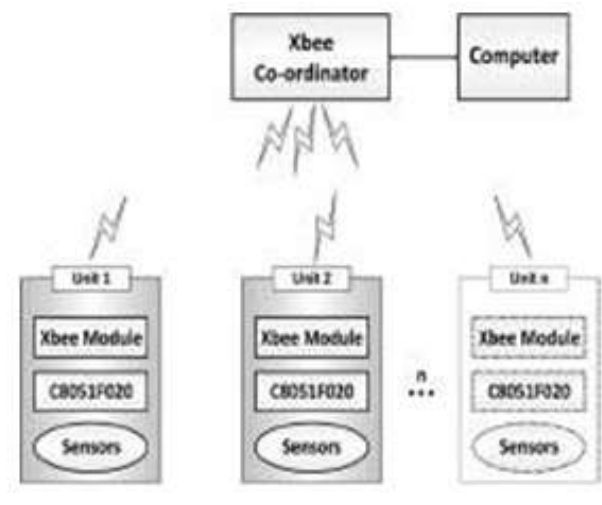

Fig. 14. System overview for wireless communication

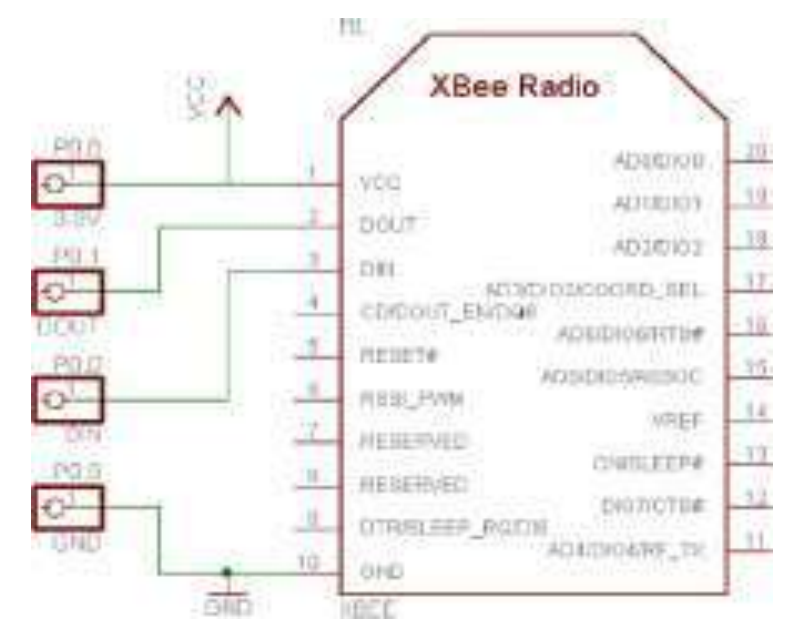

Fig. 15. XBee module electrical connection with microcontroller.

Mission. It is powered by a $9 \mathrm{~V}$ battery, and ports uses $3.3 \mathrm{~V}$, from where sensor and ZigBee modules are powered. B. XBee Module These modules provide a possibility to build an easy to configure network, with a high data rate up to $230400 \mathrm{Baud} / \mathrm{s}$. They come in a preconfigured mode and establish the communication automatically. In addition, they are powered by 2.7-3.3 V and can be connected to the C8051F020 without any additional power-supply circuit.

To connect the XBee module to the Microcontroller is done using four wires. The Power-Supply (3.3 V), Ground and TX and RX of the Microcontroller are connected to VCC, GND, DIN and DOUT of the XBee module (Fig. 15).

1) Configuration and Setup: To configure the XBee Modules, the provided software X-CTU is used. To set up a network the following conditions have to be fulfilled.

- $\quad$ Each network needs one Coordinator and several End-De vices.

- All modules have to have the same firmware and PAN-ID.

If everything is setup correct, the coordinator establishes a

connection to the End-Devices automatically.

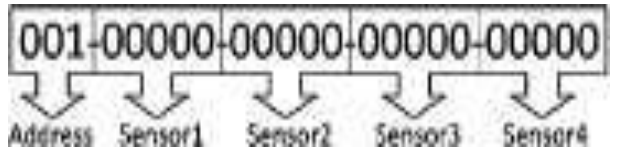

Fig. 16. Data packet composition 


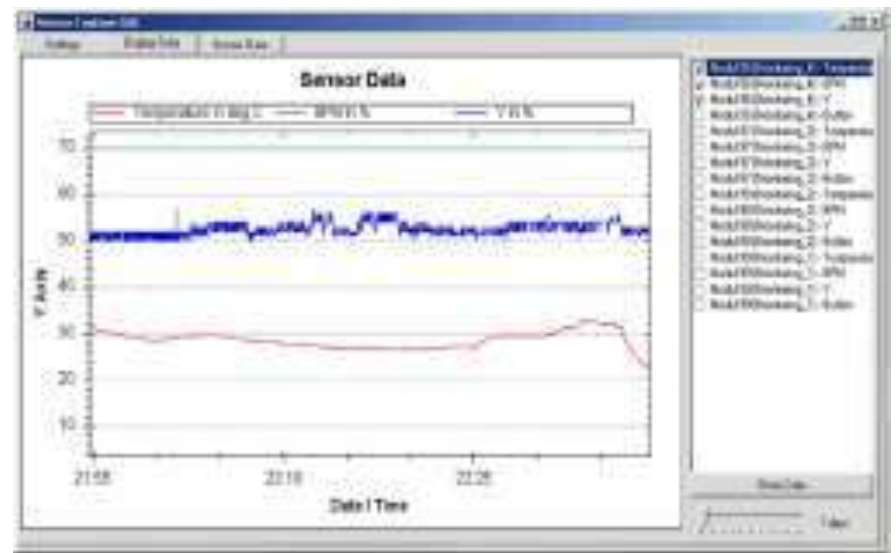

Fig. 17. Sensor data displayed from one unit/system in GUI.

The Coordinator sends Broadcast Commands, and the End-Devices can send to Coordinator only.

2) Communication Protocol: To avoid corrupted data and to see which unit was sending the data, an own communication protocol is needed. The transmission of the XBee Modules does not provide a checksum or any other possibility to verify the correctness of the received data. The send string for the sensor units contained 27 characters, as shown in Fig. 16. The first three chars are the name of the user, then each divided by a minus the sensor data. The data is raw; i.e., no processing of data is done here.

Each unit sends their data every $2 \mathrm{~s}$ to the coordinator, where the data has to be collected and tested for correctness.

Graphical User Interface (GUI): The GUI was programmed in C\# and captures the serial communication. The string received as serial Data is split into five parts (the address and sensors) and saved in an Access Database. At this stage, the GUI also tests the data for correctness.

The Database contains a Table in which the different Settings for the sensors are stored. That makes it possible to attach different sensors and to change the position of the sensor data in the send string. It also provides calibration of each sensor, for example to get the dot in the temperature. To get the right value of the sensor, sent data is divided by 100 but in the reality this value may diversify to get the right value. In the GUI, it is possible to display whether one or multiple sensors to give the user the chance to show different graphs and to compare collected data over a time period. Fig. 17 shows the data collected from one sensor unit and displayed in the computer GUI.

\section{Prototype and Experimental Results}

The SiLab microcontroller development board was used to build and test the prototype design. The analog processing circuitry and the sensors were assembled on PCBs which were placed within the wrist strap. Fig. 18 shows the prototype hardware. The prototype was powered off a $9 \mathrm{~V}$ battery. The RF transmission using ZigBee's has been tested to operate successfully at 30 meters range through obstacles such as concrete walls.

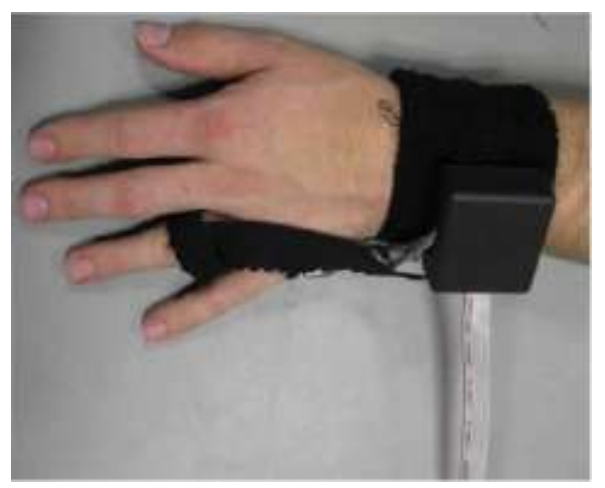

Fig. 18. Fabricated and developed prototype wrist unit 


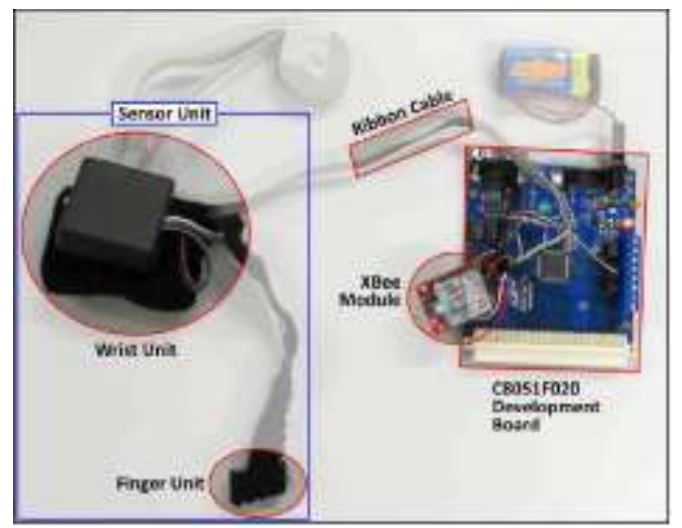

Fig. 19. Details of the prototype unit

The receiver unit can be seen in Fig. 19, without the casing. When in operation, the wrist unit consumes $20 \mathrm{~mA}$ of current at $3.3 \mathrm{~V}$ power supply, supplied from pins of a port of microcontroller. It was also recorded off DC power supply display. The microcontroller is powered by $9 \mathrm{~V}$ battery.

The XBee module connected to microcontroller consumes $40 \mathrm{~mA}$ during transmission. However, Xbee modules have the option of going in sleep mode while not transmitting. In sleep modes, XBee modules poll XBEe coordinator (their parent) every $100 \mathrm{~ms}$, while they are awake to retrieve buffered data. Pin sleep of XBee allows external microcontroller to determine when the XBee should sleep and when it should wake by controlling the Sleep_RQ pin. It saves power when no data is transmitted. By using several power-down modes that could be used to reduce consumption during times when the wrist band is not transmitting, alternatively, the architecture could be altered so that packets are only sent when a value goes outside a preset range. This was noted for future developments.

Using a $9 \mathrm{~V}$ battery which is rated at $60 \mathrm{~mA}$ for 10 hours, the device could be run continually for 25 hours before needing recharging.

\section{Discussions And Future Developments}

In this paper, we have presented the research, of applied nature, done to monitor physiological parameters such as skin temperature, heart rate, and body impact. A prototype was successfully developed and tested to establish the proof of concept. The algorithms were tested and found to be accurate and reliable at this developed/development stage. The novel aspect of the design is its low cost and detection of medical distress which does not necessitate pressing any panic button. This is an enormous improvement over existing commercial products. A panic button has also been provided in the developed system which can be used under an emergency situation.

An important aspect of the design was miniaturization, so that the system was as nonintrusive as possible to the wearer. This was achieved by the use of surface-mounted devices on the PCBs designed. Lowpower operational amplifiers were used to minimize battery consumption. The major cost comes from the use of ZigBee modules in the current design.

With some modification, the system can be made available commercially. Future improvements will focus on the use of flexible PCBs to replace the stiff cards, so that it could be moulded around the wrist unit, making it more comfortable for the wearer.

The design of the IR sensors could be improved to decrease its susceptibility to noise, to a point where it could be moved onto the wrist unit. This would provide a much more comfortable and less intrusive unit, getting rid of the need of a finger glove.

The addition of a blood-oxygen sensor would allow the system to more accurately detect medical distress by measuring the amount of oxygen in the blood $(\mathrm{HbO})$. This could be implemented by the addition of another diode operating at a different wavelength which is more readily absorbed by oxygen, and measuring the difference of absorption between the two wavelengths.

The unit was initially designed for use by the elderly, within the house, where a caregiver is present but is not able to be constantly in visual contact with the patient. The receiver unit would ideally be enhanced so that it can connect to either the local or cellular phone network, and in the case of an emergency would contact an ambulance. Beyond the application for elderly patients is the use by anyone who is at-risk, with a mental or physical disability. Monitoring of athletes whilst exercising would be possible if the sensitivity to movement was decreased. 


\section{References}

[1] J. L. Weber and F. Porotte, "Medical remote monitoring with clothes," in PHealth, Luzerne, Jan. 2006, vol. 31, pp. 246-252.

[2] J. M. Wilkinson, "Medical market for Microsystems," Int. Newsletter Microsyst. MEMS, no. 4/02, p. 37, Sep. 2002.

[3] Y. Hao and J. Foster, "Wireless sensor networks for health monitoring applications," Physiological Meas., vol. 29, no. 11, pp. R27-R56,2008.

[4] A. Pantelopoulos and N. Bourbakis, "Design of the new prognosis wearable system-prototype for health monitoring of people at risk," in Advances in Biomedical Sensing, Measurements, Instrumentation and Systems, S. C. Mukhopadhyay and A. LayEkuakille, Eds. : Springer-Verlag, 2010, vol. 55, Lecture Notes in Electrical Engineering, pp. 29-42.

[5] S. Ohta, H. Nakamoto, Y. Shinagawa, and T. Tanikawa, "A Health monitoring system for elderly people living alone," J. Telemedicine and Telecare, vol. 8, no. 3, pp. 151-156, Jun. 2002.

[6] A. Dittmar, F. Axisa, G. Delhomme, and C. Gehin, "New concepts and technologies in home care and ambulatory monitoring," Studies in Health Technol. Inform., pp. 9-35, 2004.

[7] F. Rahman, A. Kumar, G. Nagendra, and G. Sen Gupta, "Network approach for physiological parameter measurement," IEEE Trans. In-strum. Meas., vol. 54, pp. 337-346, Feb. 2005.

[8] E. Jovanov, D. Raskovic, J. Price, J. Chapman, A. Moore, and A. Krish-namurthy, "Patient monitoring using personal area networks of wireless intelligent sensors," Biomed. Sci. Instrum., pp. 373-378, 2001. [9] M. Scholtz, "Addressing the global demands for improved healthcare," in Proc. Telemedicine 21st Century, Opportunities Citizens, Society, Industry, 1999, pp. 11-18.

[10] Eastern Michigan University, "Lock-in amplification overview." [Online]. Available: http://www.physics.emich.edu/molab/lockin/index. html

[11] Products, Maxim Integrated, "DS600 \pm 0.5 accurate analog-output temperature sensor." Analog, Linear, and Mixed-Signal Devices from Maxim/Dallas Semiconductor. [Online]. Available: http://datasheets. maxim-ic.com/en/ds/DS600.pdf

[12] H. Maki, Y. Yonczawa, H. Ogawa, H. Sato, A. W. Hahn, and W. M. Caldwell, "A welfare facility resident care support system," Biomed. Sci. Instrum., pp. 480^183, 2004.

[13] Life Link Panic Button, [Online]. Available: http://www.bgehome.com/ hs_protection.html\#lifelink

[14] National Semiconductor, LM35 - Precision Centigrade Temperature Sensor. [Online]. Available: http://www.national.com/pf/LM/LM35. html

[15] Y. C. Sydney, Medical Tests. [Online]. Available: http://www.webmd. com/hw/health_guide_atoz/hw198785.asp

[16] W. D. Peterson, D. A. Skramsted, and D. E. Glumac, Piezo Film Pulse Sensor. [Online]. Available: http://www.phoenix.tcieee.org/004_Piezo_Film_Blood_Flow_Sensor/Phoenix_PiezoPulse.html

[17] K. Malhi, "Wireless sensors network based physiological parameters monitoring system," M.S. thesis, Massey University, Palmerton, New Zealand, 2010.

[18] Fall Detection Sensor System, Swiss Centre for Electronics and Mi-crotechnology (CSEM). [Online]. Available: www.csem.ch

[19] A. Lymberis, "Smart wearable systems for personalized health management: Current R\&D and future challenges," in Proc. IEEE25th Ann. Int. Conf.: EMBS, Sep. 2003, vol. 4, pp. 3716-3719.

[20] A. Gaddam, S. C. Mukhopadhyay, and G. Sengupta, "Smart home for elderly using optimized number of wireless sensors," in Advances in Wireless Sensors and Sensors Network. : Springer-Verlag, 2010, vol. 64, Lecture Notes in Electrical Engineering, pp. 307-328.

[21] V. Kremin and S. Matviyenko, "Pulse-sensing optical mouse," Circuit Cellular, vol. 194, p. $12,2006$.

[22] N. Hamza, F. Touati, and L. Khriji, "Wireless biomedical system design based on ZigBee technology for autonomous healthcare," in Proc. Int. Conf. Commun., Comput., Power (ICCCP'09), Muscat, Feb. 15-18, 2009, pp. 183-188.

[23] R. Lenhardt and D. I. Sessler, "Estimation of mean-body temperature from mean-skin and core temperature," Anesthesiology, vol. 105, no. 6, pp. 1117-1121, Dec. 2006. 